\begin{tabular}{c} 
International Journal of Engineering \& Technology, $7(3.2)(2018) 555-558$ \\
International Journal of Engineering \& Technology \\
SPC \\
Website: www.sciencepubco.com/index.php/IJET \\
Research paper \\
\hline
\end{tabular}

\title{
Research Features the Artificial Environment of Human Vital Activity
}

\author{
Valerii Usenko $^{1}{ }^{*}$, Tatyana Zinenko ${ }^{2}$, Alisa Zinenko ${ }^{3}$

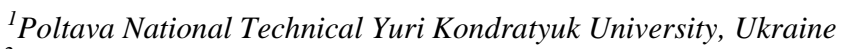 \\ ${ }^{2}$ Poltava National Technical Yuri Kondratyuk University, Ukraine \\ ${ }^{3}$ Modern Art Research Institute of the National Academy of Arts of Ukraine, Ukraine \\ *Corresponding author E-mail:valery_usenko@ukr.net
}

\begin{abstract}
Questions about the methodological difference in engineering and humanitarian approaches to the architecture study are mentioned. Attention is focused on the relevance of studying the patterns of the structure of objects in an artificial environment. Unresolved problematic issues of investigating the invariants of the architectural systems structure are considered. The inherited qualities of architectural shaping are analyzed. It is noted that ways to improve the efficiency of the system, in particular architectural, rely on the use of a system approach in research.
\end{abstract}

Keywords: Architectural system, system analysis, structural modeling, structural features of architecture

\section{Introduction}

Perceiving an object in its imagination, we give it some explanation and do not feel the difference between this phenomenon and its explanation of this object [1]. This approach also applies to architecture and other components of material and spiritual culture: design and fine arts. They constantly affect the public consciousness, really exist and depend on various manifestations of objective laws of the world. Awareness, perception, comprehension, sensation of phenomena, objects and processes accumulates experience and knowledge about the laws of their origin and existence. There are different approaches to the concepts of the object and subject of research, tasks, methods of their solution, the place and role of professionals in the construction of living space. From here is the methodological difference between engineering and humanities scienses.

\section{Problematic Questions of Architecture From the System Research Viewpoint}

The study of architecture reveals two approaches to its presentation: both a symbolic image and a system of practical use of the living environment. These approaches point to the existence of engineering and humanitarian study components of the surrounding real and spiritual world and determine the development of sciences and research methods effectiveness. Since the beginning of the Euclidean age, there has been a desire for absolute fixed-form of geometric forms in art and ture [2], [3]. A holistic understanding of the sides of the architectural systems development helps to deeply explore the subject environment and elaborate a course description of the various processes. Deepening, specialization and differentiation of certain branches of science by a number of scientists are per- ceived as a significant obstacle to the research process. The knowledge of the unity and integrity of the surrounding world must be revealed by various methods of its study. Thus, the study of complex system properties and features of artificial objects is seen as an interdisciplinary scientific direction.

An easy way to comply with the paper formatting requirements is to use this document as a template and simply type your text into it.Architectural systems are perceived as structured entities with a complex set of constraints on the concentration of various available resources. The artificial environment determines the social models of society's life, which can only operate under certain specific conditions and constraints.

Different formations exist in the real environment of the surrounding world, but they become man-made due to manifestations of human influences. Artificial environment constantly stimulates the creation and regulation of social relations: the avoidance or planning of social contacts, solitude or cohesiveness, interaction with other people and objects of its activity. To this end, important parts of the architectural system are built, linking individual actions to the sequential process. The artificial environment constantly promotes the formation of new activity varieties and provides their forms and means of existence.

Researching architecture from different points of view helps to establish its functions in human life and the factors of their changes in time, the level and influence intensity of the surrounding environment, the components dependence of its system.

Actual are the patterns study of the architectural objects structure, determined by the general natural laws. Their features are taken into account, because of numerous attempts and errors, natural selection or inherited knowledge. It is also quite conscious accumulated knowledge, which is formulated in the form of rules, norms and concepts that determine the optimal organization of artificial environment. 
There remain unresolved a number of problematic issues in the invariants study of the architectural objects structure in terms of their systematical and geometrical creation, there is no discrete nature theory of the artificial environment for the description. The structural formation concept of artificial environment objects on the basis of modern theory and practice development is not formulated. Solving the problem is required solving the following main tasks:

- finding means for formalizing the structure of the research object;

- definition of methods for analyzing the architectural systems structure.

- description of the architectural objects structure properties.

- structures systematization of artificial environment multilevel formations.

The artificial environment of different cultures (for example, ancient Greece, China and India, Byzantium, Mesopotamia and the Kingdom of Egypt) are different and have a certain number of peculiarities of their formation. An analysis of the material and civilization spiritual culture properties of proves too small possibility of a useful product numerous inventions or way of improving life as compared with the probability of its direct or indirect transmission [4].

Settlements of different cultures also have the main common features of the artificial environment organization. For example, entrances, premises, buildings, fences, streets, squares. This is due to the development of each architectural system from a single inherited community [4]. Artificial formation of the human environment manifests itself in various forms that do not have a general significant difference. This is the traditional habitation of different people: yaranga, chum, shalash, yurt, igly, vygvam, hogan, tipi (figure 1). Different types of shelter are manifested by sufficiently stable formations in space and time.
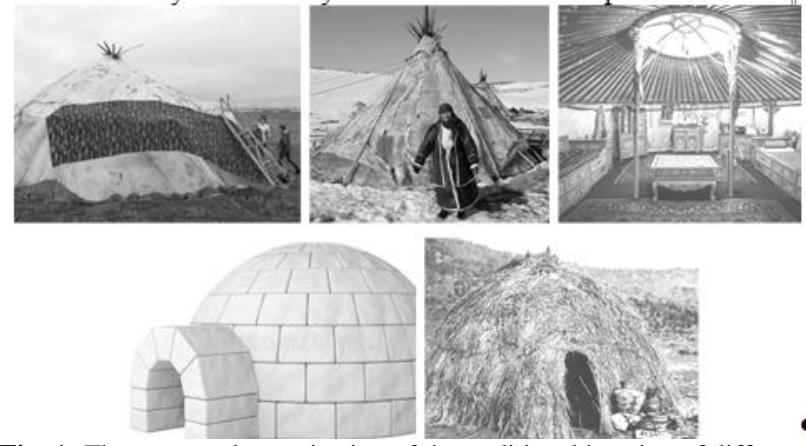

Fig. 1: The structural organization of the traditional housing of different cultures is not fundamentally different.

In the architectural and construction activities inherited qualities of people are used [5]. The qualitative property of quality appears when the human mind finds the essence of a useful and important experience for itself. Unconscious activity of people appears in the form of common achievements of previous generations architectural and construction practices. This is a generalized presentation, established algorithms of action or response in specific circumstances. They are called by necessity and take a practical image in the formation of artificial environment. The quality of construction design evolves on the basis of:

1) inherited manifestations of the truth achievement without logical justification in the construction design;

2) proven experience of previous generations and the sustainability of its norms and principles.

3) the construction of a specific shelter form, necessary and sufficient for life implementation.
In order to maintain and improve the life efficiency of different systems, it is necessary to continuously solve generalized problems of expanding and deepening the process of knowing the interaction of the system components, detecting and investigating its properties [6]. In studies it is necessary to highlight the main system concepts:

system properties:

the system is integrated into the environment;

the system may be a part of another system of a higher level generalization;

properties of its parts:

the system set of parts is adopted by a single entity;

the properties of the system differ from the properties of its parts. In figure 2 the scheme of the system generalized properties of and its constituent parts is shown.

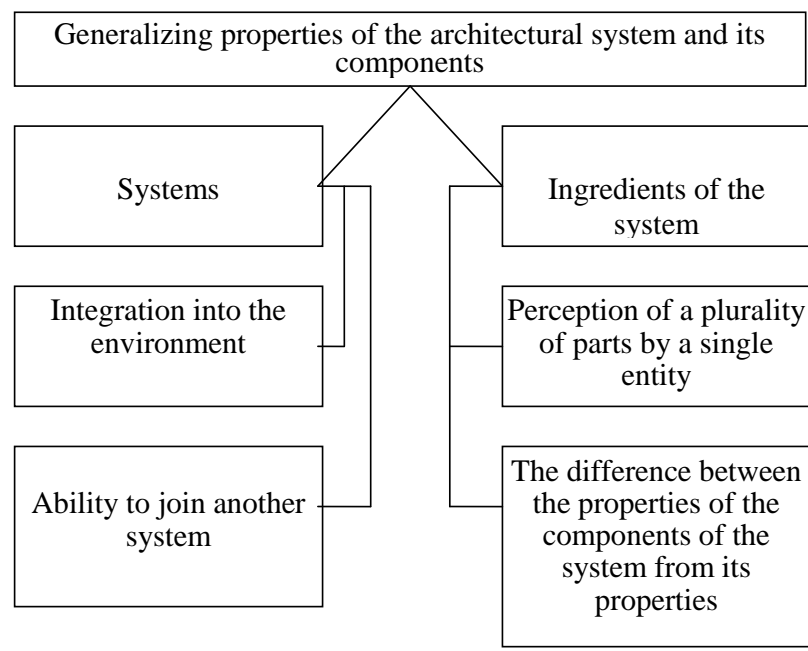

Fig. 2: Generalizing properties of the system and its components

\section{Generalizing Invariants of the Architectural System}

An important property of an architectural system is the integrity notion. It helps to separate objects from their surroundings and display them with a certain self-sufficiency. From external positions, a separate object is represented individually and independently. Inside the open and environment-related system of the integrity notion is expressed in the context of the influence of the external environment. Integrity integrates the opposite contradictory properties of the system: closed and opened. It combines the separate properties of the set of its selected parts. For example, the formation of Roman terms as a series of artificial formations with the same order of placement and the appointment of their constituent parts. However, the concept of the whole is not explained by the operation of the sum of components, because the latter are in the process of interaction within the system, but may have other specific connections to the external environment. On this basis, the concept of integrity does not become simple formalization.

Due to the systemicity, the prevalence of elements and their combinations (components) in human life, architectural education is seen as a holistic, endowed with a certain essence. The shape of any architectural object has a specific purpose. For example, some natural shelter becomes an object of artificial environment only with its acceptance by man to a number of spaces of his life. Integration of artificial formations and their isolation from the environment is one of the issues of studying the systemic properties of artificial space. For example, such objects of different scale levels and complexity such as a room, apartment, floor, house, block, urban development are often accepted as separate objects of analysis (Figure 3). The concept 
of the architectural system integrity is closely linked with the concepts of its internal unity and to a degree of autonomy.

Integrity is a condition for the existence of an architectural system, the structure of which is not in conflict with the laws of its life and the preservation of generalized properties. The system has its integrity, when its changes for a long time maintain a generalized order of their tasks execution and a stable structure.
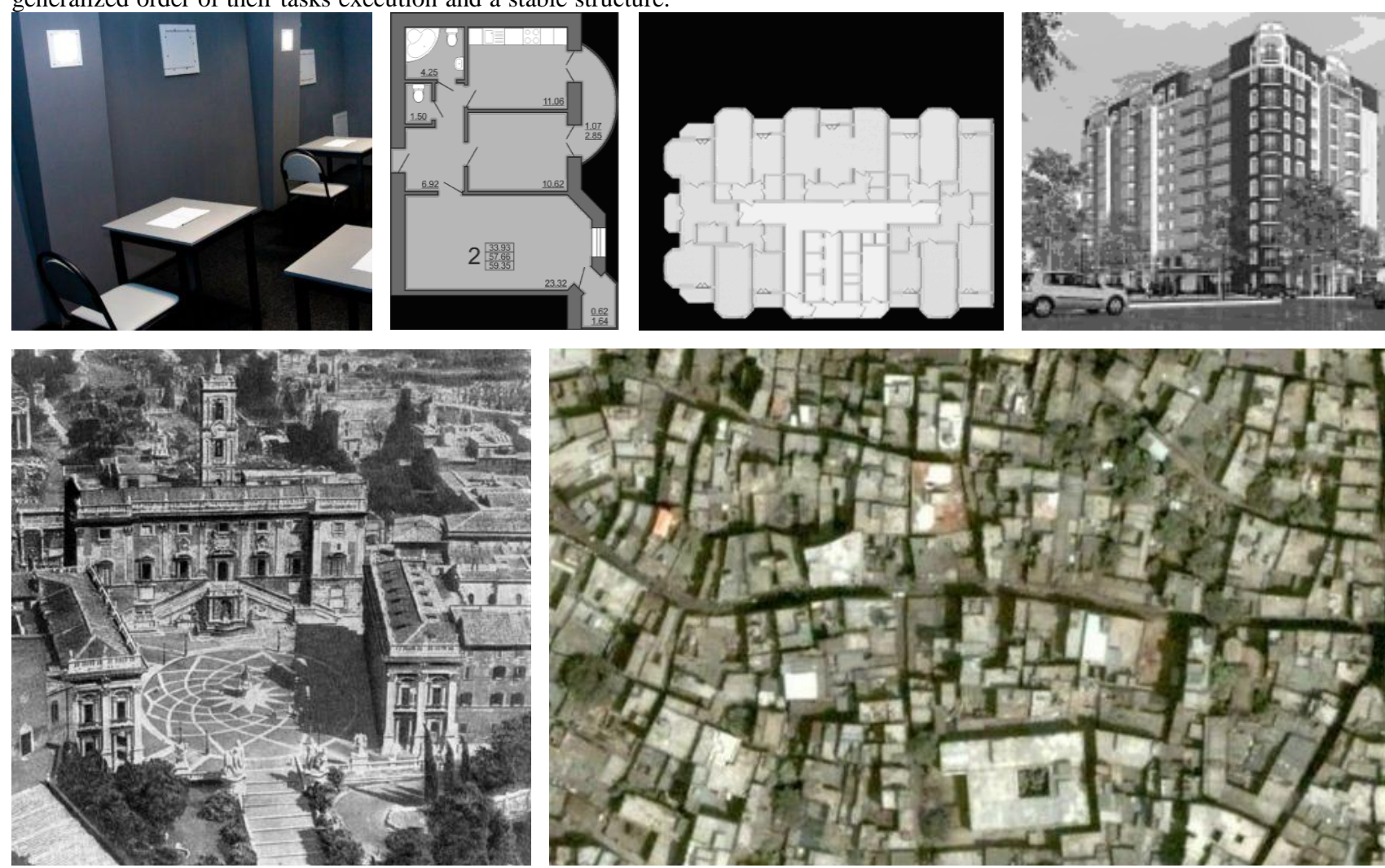

Fig. 3: Components of the architectural environment of varying complexity and scale

Architectural systems in general have certain components of invariant education: quarters, streets and squares, houses and yards, separate rooms, stairs, balconies, terraces (Figure 4). Their numerous combinations in different structural variants and forms of representation are different. This interconnection defines the architectural environment as a complex hierarchical integral system.
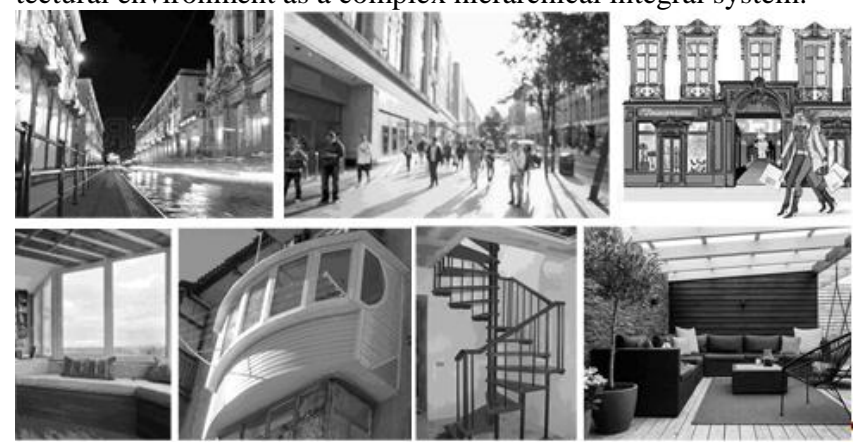

Fig. 4: Invariant formations of the architectural environment in different views

This environment is represented by a multi-level system that contains a plurality of different components (elements, subsystems) at certain complexity levels. The components of the system are linked by established relationships. In formalization, preference is given to descriptive properties of elements that they acquire in the structure of an architectural object. It is assumed that the system is dependent on all components, from their positional properties in structure relative to others. The structured set of various connected parts of the architectural system, which in certain respects, imple-
To analyze the structure and behavior of the system there are directions for studying: the whole (or system) and elements and relations [7]. ments some integrity. The constituent systems are linked together and form structural unity. In architectural form-forming, important system properties are used: component, connectivity, and integrity therefore, the system always has its generalized formalized mapping - the structure.

\section{Conclusion}

The complex definition of an architectural object important parameters today is realized by empirical methods of creating systems based on talent, skill and accumulated professionals experience.The general approach takes into account the main and important features of the system and its components. In the description with structural architectural environment and exposed to general law and omirnost objects of life from which the architectural system concept, component spine (of a combination of various difficulty levels), connectivity, integrity and functionality.

In the study of architecture, it is important to determine its functions in human life, the factors of their changes, the level and intensity of the surrounding environment influence, depending on the components of its system. Artificial environment of the human environment has clear signs of systemicity, structuring, multilevelness, complexity, multi-scale, invariance. In order to maintain and enhance the effectiveness of the architectural system, it is necessary to solve the generalized problems of expanding and deepening the interaction of its constituent parts knowing process. Investigation of an artificial sphere properties should to be realized on the basis of engineering and humanitarian approaches to the study of the surrounding real and spiritual world. 


\section{References}

[1] Kant I, Meerbote R "On the principle and form of the intelligible world", Chapter published in Theoretical Philosophy, (1755-1770), pp. 401- 405, https://doi.org/10.1017/cbo9780511840180.048

[2] Braga A Mind as Medium: Jung, McLuhan and the Archetype Philosophies Vol. 1, (2016), pp. 220-227

https://doi.org/10.3390/philosophies 1030220

[3] McLuhan M, Watson W From Cliché to Archetype, Gingko Press: Berkley, CA, USA, (2011), pp. 49

[4] Khan-Magomedov SO Dagestan labyrinths, Moscow: Ladya, (2000). pp. 11 (in Russian)

[5] Jung KG Archetype and simbol, Renaissance, Moscow, (1991), pp. 64-65

[6] Klir GJ Architecture of systems problem solving, Plenum Press, New York, (1985), pp. 22-23 https://doi.org/10.1007/978-1-44199224-6

[7] Dekkers R Applied Systems Theory, Springer, (2014), pp. 17-18 https://doi.org/ 10.1007/978-3-319-10846-9 